\title{
A 2 minute surgical hand scrub was associated with higher, but not clinically significant, bacteria counts compared with a 3 minute hand scrub
}

\author{
Wheelock SM, Lookinland S. Effect of surgical hand scrub time on subsequent bacterial growth. AORNJ 1997 Jun;65:1087-98.
}

\section{Objective}

To determine whether a 2 minute surgical hand scrub is as effective as a 3 minute surgical hand scrub for reducing bacterial growth on the hands of operating room staff.

\section{Design}

Randomised crossover study.

\section{Setting}

The operating room of a 177 bed tertiary care children's hospital in California, USA

\section{Participants}

25 staff members ( $72 \%$ women) who worked in the hospital's operating room as perioperative registered nurses $(n=21)$ or surgical technologists $(\mathrm{n}=4)$ volunteered to participate. Mean perioperative experience was 6.7 years and hand size ranged from surgical glove size 5.5 to 8 .

\section{Intervention}

Participants were allocated to 2 minute or 3 minute scrub times for a first trial, and after a minimum of 7 days were allocated to the alternative scrub time. Participants, under the supervision of the study investigators, scrubbed at sinks equipped with timers, and used a scrub protocol based on existing guidelines by the Association of Operating Room Nurses, the American Society for Testing and Materials, and the US Food and Drug Administration. 10 participants used 4\% chlorhexidine gluconate (CHG) as a scrub agent, and 15 participants who had previously experienced skin reactions used scrub agents that were less irritating (eg, 2\% CHG or parachlorometaxylenol). Participants then put on sterile surgical gloves and completed their regular duties, but had no patient or surgical field contact.

\section{Main outcome measures}

Bacterial growth under the surgical gloves was measured using the "glove juice sampling" procedure. After participants wore the gloves for 1 hour, $50 \mathrm{ml}$ of sterile solution was introduced into the glove of the participant's non-dominant hand; the glove was then secured at the wrist with a rubber band, and the participant vigorously rubbed the hand for 1 minute. Sample dilutions of $0.5 \mathrm{ml}, 0.1 \mathrm{ml}$, and $0.05 \mathrm{ml}$ were plated and incubated for 48 hours. Colony forming units were counted by hand, averaged for plate counts, and converted into $\log _{10}$ values.

\section{Main results}

The mean log colony forming unit count was higher for the 2 minute scrub (4.23 log) compared with the 3 minute scrub (3.94 log). The log standard error of the mean difference between groups was $0.29(\mathrm{p}=0.02)$. Although the mean bacterial count differed statistically, the difference fell below the threshold for practical and clinical significance $(0.5$ log standard error of the mean difference between groups). The glove perforation rate was $4 \%$.

\section{Conclusion}

A 2 minute surgical hand scrub was associated with higher, but not clinically significant, bacterial growth on the hands of operating room staff compared with a 3 minute surgical hand scrub.

Source of funding: not stated.

For article reprint:Dr S Lookinland, Community Health Systems, Fresno, CA, USA. Fax +1 209266 3876.

\section{Commentary}

The surgical hand scrub is a time honoured ritual, integrated into the practice of the perioperative healthcare provider. The rationale for this practice is grounded in the belief that a prescribed surgical hand scrub technique decreases the growth of microorganisms and prevents wound infections. Standards of practice for the surgical hand scrub are based on limited and inconclusive research and tradition. Current surgical hand scrub protocols have also increased the incidence of dermatitis in practitioners and put into question current practices.

The study by Wheelock and Lookinland provides empirical evidence to challenge the 3 minute surgical hand scrub and suggests reform of this practice might be necessary. The experimental, ran- domised, crossover design explored differences between 2 and 3 minute surgical hand scrub procedures and measured bacterial growth under the surgical gloves. The results of this investigation indicated higher bacterial growth on the hands of operating room staff using the 2 minute as compared with the 3 minute hand scrub. The authors believe that these results fall below the accepted threshold for clinical and practical significance.

The authors reported that $84 \%$ of the total sample $(n=25)$ were women, with smaller hand sizes than the men in the study. Generalisability could be improved by studying a larger number of staff including more male operating room staff. Future studies across several sites with analysis of multiple variables (eg, sex, hand size, hand scrub time, wound infection, and hand scrub solution) could increase the reliability and validity of study findings. Other factors such as alternate work patterns for operating room staff, which would reduce the number of continuous days an individual did hand scrubs, may affect the incidence of dermatitis.

Continued investigation is needed to change standards of surgical hand scrub practice.

Dorothy A Jones, RNC, EdD, FAAN
Nurse Scientist
Massachusetts General Hospital
Chairperson
Adult Health, Boston College
Boston, Massachusetts, USA

